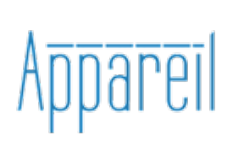

Appareil

$6 \mid 2010$

Philosophie et cinéma

\title{
Les spectres de Raúl Ruiz. La maison Nucingen (2009)
}

Adolfo Vera

\section{OpenEdition}

1 Journals

\section{Édition électronique}

URL : http://journals.openedition.org/appareil/934

DOI : 10.4000/appareil.934

ISSN : 2101-0714

Éditeur

MSH Paris Nord

Référence électronique

Adolfo Vera, "Les spectres de Raúl Ruiz. La maison Nucingen (2009) », Appareil [En ligne], 6 | 2010, mis en ligne le 10 février 2010, consulté le 30 juillet 2020. URL : http://journals.openedition.org/ appareil/934; DOI : https://doi.org/10.4000/appareil.934

Ce document a été généré automatiquement le 30 juillet 2020.

\section{(c) (i) () $९$}

Appareil est mis à disposition selon les termes de la Licence Creative Commons Attribution - Pas d'Utilisation Commerciale - Pas de Modification 4.0 International. 


\title{
Les spectres de Raúl Ruiz. La maison Nucingen (2009)
}

\author{
Adolfo Vera
}

1 Toute l'œuvre cinématographique (plus d'une centaine de films) de Raúl Ruiz (Chili, 1941) est traversée par la question de la spectralité. Peu de cinéastes ont exploré, comme lui, les possibilités techniques propres à l'image cinématographique pour créer et représenter ce qu'on pourrait appeler la « réalité des spectres ». Cette réalité, dans l'œuvre de Ruiz - depuis la Vocation suspendue jusqu'à La maison Nucingen - est puissamment déterminée par l'usage inouï qu'il fait des possibilités techniques propres à l'appareil cinématographique. Il s'agit, pour Ruiz, d'une exploration systématique de ce que nous appellerons les «possibilités spectrales» de l'image cinématographique. Tenant compte de sa célèbre thèse contre «l'idéologie du conflit central » - c'est-àdire, contre l'idée qu'un film doit raconter une histoire qui tourne autour d'un sujet, à la manière aristotélicienne, avec des personnages qui persistent dans la trame narrative et en permettent le développement, tout en déterminant aussi les autres aspects techniques et discursifs du film -, le cinéaste travaille avant tout à partir de l'usage particulier qu'il fait de moments techniques essentiels à la réalité du film comme à celle du montage, de la bande son et du scénario (qui va perdre son importance par rapport aux deux premiers éléments). L'œuvre de Ruiz a insisté sur la configuration de la réalité du film comme une totalité ouverte et toujours en expansion (dans ce sens-là presque une anti-totalité), totalité dans laquelle aucun moment n'est plus important qu'un autre, chacun d'entre eux tendant à une dislocation constante de l'unité du film. Tout ceci donnera lieu à la considération de l'œuvre filmique comme une totalité non-homogène et non unitaire (anti-totalité) : Ruiz s'appuie pour cela sur des théories de la physique quantique - on pourrait dire que l'un de ses apports le plus importants est justement d'avoir installé un «principe d'incertitude» dans le récit cinématographique - et sur celles de la neuroscience, dans le sens de la définition de la pensée par «structures émergentes » chez Francisco Varela : la création de la réalité par la pensée se constitue à partir de noyaux de sens qu'aucune objectivité ne donne à l'avance. En même temps, cela va permettre (et des films comme L'hypothèse du tableau volé et Les trois couronnes du matelot, Días de campo (Les jours dans la campagne) et Trois vies 
et une seule mort sont essentiels dans ce sens-là) l'élaboration d'une réalité (la réalité du film) qui possède ses propres lois, autonomes par rapport à la réalité objective, et qui se développe à partir d'une spatio-temporalité qui ne cherche pas à se connecter avec le «monde» extérieur au cinéma. Bien que de nombreux critiques l'aient taxé d'« esthétisme » - péché majeur pour un cinéaste latino-américain -, Ruiz semble nous dire (et en fait c'est l'un des sujets importants de son livre La poétique du cinéma $a^{1}$ ) que toute critique sociale et politique - on verra plus tard comment la relier à la question de la spectralité - apparaîtra comme inhérente au langage cinématographique, strictement particulière à l'univers du film, univers toujours régi par les lois de l'appareil cinématographique.

2 Qu'est-ce qu'un spectre? On peut répondre de diverses manières. Dès la réponse proprement «spiritiste»-celle que l'on méprise d'habitude, mais qui plonge ses racines dans les profondeurs des croyances humaines, et qui a été, par exemple, la position défendue par Schopenhauer dans son Essai sur les fantômes ${ }^{2}$ - une certaine définition s'ébauche qui se distingue de celles de la philosophie et de la science : elle est fondée sur la croyance dans des esprits, des entités déambulant entre la vie et la mort et qui « reviennent » pour nous hanter ${ }^{3}$; Freud rattache une telle mythologie à la notion de l'inquiétante étrangetét. D'autre part, la philosophie et la science essaient de prouver l'existence de ces " esprits » en les soumettant à l'épreuve d'une objectivation empirique et technologique, notamment celle de l'appareil photographique. En vérité, chacun de ces « regards »- puisque il s'agit là d'apparitions et de disparitions - cherche à fusionner avec celui de la mythologie ésotérique des esprits. De la même manière, les expérimentations «scientifiques » que faisaient Bergson et Marey, au début du siècle dernier, pour démontrer que l'appareil photographique pouvait capturer les « spectres » - c'est-à-dire, les émanations d'« éther » des êtres vivants - ont des rapports évidents avec ces conceptions dites « mythologiques $^{5}$ ».

3 Dans le contexte de ce genre de pratiques, la culture européenne a reliée dans son approche de la question des spectres, les modalités de production de l'appareil photographique et cinématographique, à un régime «spectral » de leurs images. Nadar, dans ses mémoires intitulées Quand j'étais photographe ${ }^{6}$, raconte comment Balzac, dans la décennie suivant la découverte de la procédure photographique par Niepce, avait élaboré une théorie qu'il appelait «Théorie des spectres ${ }^{7}$ ». Selon Balzac, la photographie pourra capturer les "spectres", et il en ressort toute une série de questions qui empruntent à différentes sources philosophiques, "scientifiques » et littéraires, comme celle du "magnétisme animal», noyau dur du texte de Schopenhauer déjà mentionné. D'après ces sources, on en arrive à la conclusion qu'il y a des énergies que l'œil ne peut pas voir, même si elles font partie de la "vie animale » et que, peut-être, la photographie pourra "capturer». Ces énergies seront appelées «spectrales », et Balzac osera même postuler que chaque photo prise d'un être vivant impliquera la saisie d'une couche de cette énergie, et son affaiblissement progressif; une extension de cette théorie - développée par quelques physiologues de l'époque ${ }^{8}$, aboutit à ce que la vie d'un être humain se résume à la correspondance avec une quantité déterminée et finie d'énergie. Plus cet être dépense son énergie, moins il vivra ; ce thème sera aussi au cœur de son fameux roman, La peau de chagrin, où l'on voit le protagoniste recevoir d'un vieil antiquaire un talisman (un morceau de la peau de chagrin) qui pourrait accomplir tous les desseins du jeune homme, sauf que chaque dessein accompli impliquera la réduction du morceau de peau, et, par suite, de la vie du 
jeune homme. Ce n'est pas seulement une coïncidence si La maison Nucingen, le dernier film de Raúl Ruiz que nous allons commenter, est basé sur une nouvelle éponyme de Balzac. Depuis lors, et jusqu'à Anton Bragaglia, photographe futuriste italien qui pratiquait systématiquement la "photographie de fantômes ", la photographie, l'art et le monde des spectres ont scellé un pacte étroit, pacte qui s'étend aussi aux usages dits sociaux de la photographie, usages qui indiquent la croyance profonde - propre à chacun d'entre nous - que ces pigments ou pixels se réfèrent à une existence "réelle et concrète ». À partir de Derrida, on pourra dire que la question de la référence, et donc du nom, apparaît inséparable de la question de la spectralité ${ }^{9}$. Ces images possèderaient la réalité au même niveau que ce qui a été photographié, ce que la "stricte rationalité " oblige à nier. En attribuant à ces images (dès lors transformées en «simulacres ", en "fantasmes ») une vie propre, dès lors qu'il s'agit de celles de nos chers défunts, on se prépare à les associer à un rite mortuaire. C'est dans ce sens que Benjamin parlait de la "valeur cultuelle» de la photographie; chaque photo, en plus du moment technique lui aussi essentiel qui définit sa reproductibilité, renvoie aux conceptions les plus archaïques de l'image, le portrait photographique étant, selon Benjamin, le dernier endroit où se réfugie cette " valeur cultuelle ».

4 Il est évident que, dans ce sens, les considérations de Benjamin permettent d'observer comment dans «l'image technique » s'infiltrent des croyances qui, dans un premier abord, paraissaient complètement étrangères à de pareilles productions de la technologie. C'est de cette manière que la croyance aux spectres, plus précisément dans la possibilité d'observer directement ces êtres normalement imperceptibles, pouvait s'originer dans les possibilités techniques de l'appareil photographique, appareil dont Flusser établit que l'approche la plus "normale » - même pour le photographe - est l'approche "magique ${ }^{10}$ ». Or, l'idée centrale, dans ce sens, est celle selon laquelle l'appareil photographique pourrait capturer des réalités que l'œil humain ne peut pas voir. C'est justement cela que Benjamin appelait «l'inconscient optique ».

5 Après «l'image technique " propre à la photographie, l'image cinématographique acquerra la même possibilité d'accéder à la matérialité imaginale des spectres. Dans un contexte plus complexe, pas seulement en ce qui concerne les conditions techniques particulières au cinéma - notamment celles de la constitution de l'image-mouvement, de la possibilité de la bande son et de la conformation globale de ces éléments grâce au montage -, mais aussi en terme de production et d'industrialisation, le cinéma se transformera en une véritable usine de spectres: tout cela (il faudra ajouter la télévision et la vidéo) permettra à Jacques Derrida d'affirmer que «l'avenir appartient aux fantômes », puisque l'avenir est ce que, lui, appelle le « techno-télé-pouvoir ${ }^{11}$ ».

Toutes ces questions peuvent se retrouver dans les films de Raúl Ruiz, en particulier dans le tout dernier, La maison Nucingen.

Comme dans la plupart de films de Ruiz - et ici on peut songer aux Trois couronnes $d u$ matelot (France - Chili, 1983), ou aux Trois vies et une seule mort (France - Portugal, 1995), mais aussi à Días de campo (Les jours dans la campagne, France-Chili, 2004) ou à la série réalisée pour la télévision publique chilienne La recta provincia (La province droite, Chili, 2007), dans La maison Nucingen (Roumanie-France-Chili, 2009), la question de la réalité des spectres est centrale. Dans chacun de ces films, il s'agit d'indiquer l'impossibilité - à l'intérieur du monde autonome du film - de différencier les "spectres» des "êtres vivants " (devenus des spectres cinématographiques, au moins aussi vivants que les vivants). On pourrait dire que, à proprement parler, chaque objet filmé apparait, dans 
la projection, comme un fantasme (et ici le rapport étymologique entre "fantasme", "fantôme " et "simulacre " est très instructif ${ }^{12}$ ) puisque finalement il ne s'agit que d'illusions ; en tant qu'image, un tel objet ne possède pas une réalité semblable à celle qu'on trouve dans la « réalité »; comme chez Platon, l'image est la « copie d'une copie » (l'objet réel étant lui-même la copie de l'Idée), c'est-à-dire, un simulacre, un fantasme. Cela étant correct, il ne faut pas oublier que la «bataille » de Ruiz se mène justement contre ce qu'il appelle "l'idéologie du conflit central ", qu'on peut résumer comme le postulat (aristotélicien) selon lequel l'œuvre cinématographique (comme celles du théâtre et de la littérature classiques) doit "donner l'illusion» (mimesis) que ce que le spectateur observe est une version formalisée par les « règles de l'art » de la « réalité objective » la souffrance d'une femme qui a appris la mort par suicide de son fils est la souffrance d'une « femme réelle » et non celle d'une comédienne entourée de caméras, d'appareils divers, et de dizaines d'opérateurs - justement pour y produire le pathos. Cette conception « rétrograde » de l'art - par rapport au théâtre et à la littérature qui, depuis Flaubert et Brecht au moins, avaient déjà abandonné Aristote - s'est imposée très vite dans l'industrie américaine, même si le cinéma européen tendait vers le contraire. En fin de compte, la révolte contre cette vision du cinéma est caractéristique de la plupart des œuvres des cinéastes qui ont influencé Raúl Ruiz: Godard, Antonioni, Buñuel, Duras, Glauber Rocha, Pasolini ou Bergman. Chez Ruiz, il s'agit donc de montrer (comme on l'a déjà dit, grâce aux possibilités techniques de l'appareil cinématographique) comment, dans l'univers du film, il est impossible de distinguer entre « êtres vivants » et « revenants » ou "fantômes ». Or, dans Les trois couronnes du matelot, l'image diffuse et floue, avec des plans en contre-plongée trop marquée et complètement anti-naturels, renforce la conscience de l'artifice à un montage aléatoire et non déterminé par la progression d'un "récit ", permettant l'élaboration d'une atmosphère spectrale. Dans le cas de Días de campo, il s'agissait notamment de la photographie des intérieurs d'une ancienne maison de la campagne chilienne, des espaces sombres et comme au-delà du temps; dans La maison Nucingen - comme aussi dans L'hypothèse du tableau volé, Klimt ou dans Trois vies et une seule mort - «l'effet de spectralité » (Derrida) apparaît à partir de l'élaboration d'un phénomène particulier au récit (une "fable», pourrait-on dire avec Rancière) que nous identifierons avec la " rumeur » et non pas avec le " discours » et ceci grâce à l'utilisation singulière que fait Ruiz des possibilités de la bande-son - voix off, musique, bruits, "discours" des personnages - qui y introduisent une atmosphère de confusion et d'irréalité par rapport à l'attente d'une histoire linéaire et univoque : il se trouve que dans les films de Ruiz - notamment dans le tout dernier, La maison Nucingen - on ne s'installe pas dans le registre de la " communication » mais on évolue dans celui de la « rumeur ", un monde composé d'une intrication de phrases, de fragments de discours, de voix, de bruits qui, en vérité, traduisent un éclatement de la temporalité et un anachronisme qui, selon Derrida, constitue le noyau de ce que lui-même appelle "l'effet de spectralité ${ }^{13}$ ». Il signifie la confusion et la dissolution de l'ordre des catégories logiques qui déterminent habituellement la progression d'une histoire et génèrent la confusion entre les êtres de fiction cinématographiques et les êtres vivants de chair et de sang. L'essence de l'image filmique étant la production de spectres, le cinéaste, en soulignant ce principe, montre la puissance manipulatrice de cette fonction de spectralité qui relève du fonctionnement intrinsèque de l'appareil cinématographique.

8 Le récit (pour ne pas dire « l'argument » puisqu'il est possible au cinéma de parler d'un récit sans «argument») de La maison Nucingen est simple, et concerne le genre, déjà 
classique, des histoires de vampires, dont le référent le plus important, en ce qui concerne la littérature, comme on sait, est le roman Dracula (1897) de Bram Stoker, et au cinéma le Nosferatu (1922) de Murnau, l'un des chef-d'œuvres de l'expressionnisme allemand. En fait, le film de Ruiz est basé sur la nouvelle éponyme de Balzac et sur un récit de Mircea Eliade, le célèbre historien des religions roumain. Évidemment, le film de Ruiz s'inspire plutôt du récit de vampires du mythologue que de la nouvelle réaliste de Balzac, et fait surgir le récit du film d'un mélange entre les deux : un aristocrate obtient une vielle maison en jouant au poker, et il part ensuite avec son épouse pour y habiter. La maison - là, bien entendu, c'est Ruiz qui ajoute - est située au Chili, et le couple s'embarque pour un long voyage. Quand ils arrivent, ils sont reçus par les proches de l'ancien propriétaire qui habitent là-bas. Tout de suite, le couple note qu'il y a «des esprits » dans la maison, et à la question : «combien de personnes habitent ici?", posée par le mari, l'homme qui les reçoit répond: «Je ne sais pas vraiment, peut-être dix, cinquante, cent...»; les habitants de la maison semblent donc bien habitués à ces présences inquiétantes. La maison apparaîtra alors comme un univers autonome, comparable à celui du cinéma, dont les lois sont les lois de l'appareil cinématographique, et non pas celles de la « réalité ». Ces lois, comme on l'a déjà dit, sont foncièrement spectrales.

9 Le récit va donc se fonder sur les options techniques de Ruiz, depuis la plus simple comme l'est celle de filmer en DV (format qui oblige à la perception de se concentrer dans des tonalités froides, par lesquelles le film apparait surchargé d'une atmosphère glaciale, cadavérique) jusqu'à l'utilisation de la voix off se référant à des faits sans rapport avec ce qui se passe dans l'action; de plus, la bande-son (ceci est une constante du travail de Ruiz) est hantée par les chants d'une autre époque, par des voix d'enfants, et aussi par des bruits d'objets hors champ : tout cela en ayant pour but la propagation des "effets de spectralité », c'est-à-dire des "effets d'anachronisme ", soulignant la "non-contemporanéité du présent avec lui-même ». L'anachronisme majeur, bien sûr, est celui d'avoir choisi une vieille maison du Chili pour scénographie, maison qui - en considérant tous les indices du « récit » - devrait être une maison de la fin du XIX ou du début $d u x^{e}$, mais qui nous apparaît comme une vieille maison «aujourd'hui »- dans ce présent écrasé et ouvert à toutes les temporalités - habitée par des sujets habillés selon une autre époque. Ces aspects, en fait, ont été fortement critiqués par certains critiques qui ont considéré que ces "manques de coordination"-entre le son et l'image, entre l'attitude des comédiens et le « sens » du discours qu'ils profèrent, etc. obéissaient à des « erreurs » - même si Ruiz parle depuis longtemps d'une " poétique de l'erreur »-, quand tout cela est là justement en tant qu'« effet de spectralité ».

Effet, aussi, politique de la spectralité comme puissance anachronique : le Chili n'est-il pas un pays où, justement, après tout le sang versé dans les rues, après tant de disparus devenus aujourd'hui nos véritables spectres revenant nous hanter, après tout cela, le Chili n'est-il pas un pays qui, comme La maison Nucingen, est toujours hanté quotidiennement par l'absence de communication, l'impossibilité de vivre au présent et d'identifier le temps qui lui est propre? Un pays de fantômes et de vampires? 


\section{BIBLIOGRAPHIE}

Braun Marta, «Fantasmes de vivants et des morts », Études photographiques, 1, novembre 1996, [En ligne], mise en ligne le 18 novembre 2002. URL: http://etudesphotographiques.revues.org/100. Consulté le 24 septembre 2009.

Citron Pierre, Introduction à La peau de chagrin, Paris, Garnier Flammarion, 1971.

Déotte Jean-Louis, L'époque des appareils, Paris, Lignes \& Manifeste, 2003.

Derrida Jacques, Saufle nom, Paris, Galilée, 1993.

Derrida Jacques, Spectres de Marx, Paris, Galilée, 1993.

Fabry Jacques, Visions de l'au-delà et tables tournantes, Paris, Presses universitaires de Vincennes, 2009.

Flusser Vilèm, Pour une philosophie de la photographie, Paris, Circé, 2004.

Freud Sigmund, L'inquiétante étrangeté et autres essais, Paris, Gallimard, 1985.

Krauss Rosalind, Le photographique. Pour une théorie des écarts, Paris, Macula, 2000.

Nadar, «Quand j'étais photographe », in Dessins et écrits, t. II, Paris, Arthur Hubschmid, 1979.

Ruiz Raúl, Poétique du cinéma 1, Paris, Dis Voir, 1995.

Ruiz Raúl, Poétique du cinéma 2, Paris, Dis Voir, 2006.

Schopenhauer Arthur, Essai sur les fantômes, Paris, Criterion, 1992.

\section{NOTES}

1. Raúl Ruiz, Poétique du cinéma 1, Paris, Dis Voir, 1995 ; Poétique du cinéma 2, Paris, Dis Voir, 2006.

2. Arthur Schopenhauer, Essai sur les fantômes, Paris, Criterion, 1992.

3. Jacques Fabry, Visions de l'au-delà et tables tournantes, Paris, Presses universitaires de Vincennes, 2009.

4. Sigmund Freud, L'inquiétante étrangeté et autres essais, Paris, Gallimard, 1985.

5. Marta Braun, «Fantasmes de vivants et des morts », Études photographiques, 1, novembre 1996, [En ligne], mise en ligne le 18 novembre 2002. URL: http://etudesphotographiques.revues.org/ 100. Consulté le 24 septembre 2009.

6. Nadar, «Quand j'étais photographe », in Dessins et écrits, t. II, Paris, Arthur Hubschmid, 1979.

7. Cf. aussi Rosalind Krauss, Le photographique. Pour une théorie des écarts, Paris, Macula, 2000.

8. Cf. l'Introduction à La peau de chagrin, Paris, Garnier Flammarion, 1971, par Pierre Citron, où sont cités les noms de Hyacinthe Azaïs et de Ballanche, auteur d'une Palingénésie sociale.

9. Cf. Jacques Derrida, Saufle nom, Paris, Galilée, 1993 ; Spectres de Marx, Paris, Galilée, 1993.

10. Cf. Vilèm Flusser, Pour une philosophie de la photographie, Paris, Circé, 2004.

11. Cf. Jacques Derrida, Spectres de Marx. Derrida affirme - mais on ne peut pas insister là-dessous ici - que tout événement, en étant toujours déterminé par ce qu'on ne pouvait pas prévoir, et alors par l'ouverture absolue de la possibilité de l'arrivée de l'imprévisible, est essentiellement spectral. Pour une critique de cette conception, à partir de la question de la disparition politique, cf. Jean-Louis Déotte, L'époque des appareils, Paris, Lignes \& Manifeste, 2003.

12. L'entrée pour «Fantasme » du Dictionnaire étymologique de la langue française, Paris, Le Robert, 1992, indique : « n. m. est emprunté au latin impérial phantasma "fantôme, spectre", en bas latin 
“image, représentation par l'imagination", transcription du grec phantasma "apparition, vision, fantôme", de la famille de phainein "apparaître" (fantaisie) [...] ». Pour l'entrée " Fantôme », nous est indiqué: « ...Fantôme est introduit en français avec le sens d'“illusion trompeuse", qui existe déjà en latin, et désigne (1165) l'apparition surnaturelle d'une personne morte ; par extension, le mot s'emploie pour "idée, être imaginaire" (1586) et à propos d'une personne ou d'un animal squelettique (1690) [...]». Op. cit., t. I, 778-779. L'entrée pour «Simulacre» indique: «n.m. réfection graphique de simulacre (v. 1170), est un emprunt au latin simulacrum "représentation figurée (de qqch.) " d'où "image, portrait, effigie, statue" et par figure "fantôme, ombre", "apparence". Le mot signifie spécialement dans la langue philosophique "image, simulacre des objets", par traduction du grec eidolon (->idole). Simulacrum dérive de simulacre "rendre semblable", d'où "feindre" (simuler) ». Op. cit., t. II, p. 1948.

13. Jacques Derrida, Spectres de Marx.

\section{RÉSUMÉS}

Raúl Ruiz, cinéaste chilien exilé en France à la fin des années 1970, a construit une œuvre très vaste et donc très difficile à comprendre univoquement. La critique (un numéro spécial lui était consacré par les Cahiers du cinéma en 1983) a depuis toujours signalé l'absence d'un récit net qui organise ses films, la multiplicité des idées qui s'ouvrent en chaque plan (« chaque idée un plan, chaque plan un film », a postulé un jour Ruiz), la teneur "baroque » d'une œuvre où les limites entre le réel et l'irréel se brouillent du fait des particularités techniques de l'appareil cinématographique. Dans ce texte, on propose une lecture des quelques aspects de cette œuvre inabordable à partir de la question de la spectralité, question qui, selon Derrida (Spectres de Marx) constitue le noyau de la situation politique contemporaine. C'est ce que Derrida appelle "puissance de spectralité», et qui se manifeste notamment en tant que "puissance d'anachronisme ", ce qui inonde les images cinématographiques de Ruiz, pour créer un univers où les moyens techniques du cinéma reprennent depuis son origine, au XIX $\mathrm{e}^{\mathrm{e}}$, les «images techniques " photographiques et cinématographiques étaient censées constituer : l'apparition des fantômes. Or, on sait bien que, depuis la stratégie de la disparition politique appliquée par tous les totalitarismes $\mathrm{du} \mathrm{xx}^{\mathrm{e}}$ siècle et d'aujourd'hui, la catégorie de «fantôme » a bien un aspect politique. Le dernier film de Ruiz, La maison Nucingen (2009) met toutes ces questions en lumière.

\section{INDEX}

Mots-clés : fantôme, appareil, cinéma, disparition, spectralité, spectre

Personnes citées : Ruiz (Raúl), Derrida (Jacques) 
AUTEUR

ADOLFO VERA

Docteur en Philosophie université Paris VIII Vincennes Saint-Denis. Professeur Institut de Philosophie université de Valparaíso, Chili. 